\title{
Effets de la rhizobactérie Bacillus amyloliquefaciens FZB 42 sur la tolérance au stress hydrique et le rendement du cotonnier
}

\author{
B. C. Thiery ALAVO ${ }^{1 *}$, Malick ABOUdOU ${ }^{1}$, Luc DIDOLANVI ${ }^{1}$ et G. Dossou FAYALO ${ }^{2}$ \\ ${ }^{I}$ Faculté des Sciences et Techniques, Université d'Abomey-Calavi, BP 215 Godomey, Bénin. \\ ${ }^{2}$ Centre de Recherches Agricoles - Coton et Fibres (CRA-CF), Institut National des Recherches Agricoles du \\ Bénin (INRAB), Bénin. \\ *Auteur correspondant ; E-mail: thieryalavo@hotmail.com
}

\section{RESUME}

Le présent travail a évalué les potentialités de Bacillus amyloliquefaciens FZB 42 pour la production cotonnière. Pour ce faire, l'effet de la rhizobactérie sur la tolérance au stress hydrique et le rendement du cotonnier a été étudié. Pour déterminer si la rhizobactérie peut permettre au cotonnier de résister au stress hydrique, 4 variantes ont été testées, à savoir : i) semences traitées avec la rhizobactérie avec arrosage d'eau pendant 15 jours, ii) semences traitées avec la rhizobactérie sans arrosage, iii) semences non traitées avec la rhizobactérie, avec arrosage d'eau, et iv) semences non traitées avec la rhizobactérie, sans arrosage. Pour tester l'effet sur le rendement du cotonnier, 6 traitements ont été mis en comparaison, à savoir : i) Semences non traitées avec la rhizobactérie et fertilisation avec une dose pleine d'engrais minéraux, ii) Semences traitées avec la rhizobactérie sans apport d'engrais minéraux, iii) Semences traitées avec la rhizobactérie avec apport de $10 \%$ d'engrais minéraux, iv) Semences traitées avec la rhizobactérie avec apport de $25 \%$ d'engrais minéraux, v) Semences traitées avec la rhizobactérie avec apport de $40 \%$ d'engrais minéraux, et vi) Semences traitées avec la rhizobactérie avec apport de $50 \%$ d'engrais minéraux. Les résultats ont montré que les semences traitées avec B. amyloliquefaciens FZB42 ont germé en abondance et plus vite que les semences non traitées, et les plantules issues d'elles se sont développées plus rapidement que celles du témoin (non traitées), quelles que soient les conditions de stress hydrique. Les cotonniers traités avec la rhizobactérie puis fertilisés avec $40 \%$ ou $50 \%$ de la dose d'engrais minéraux recommandée ont donné un rendement similaire à celui des plants ayant reçu la dose pleine d'engrais. La rhizobactérie B. amyloliquefaciens FZB42 peut être intégrée dans le système de production cotonnière dans le but de réduire la dépendance aux engrais minéraux.

(C) 2018 International Formulae Group. All rights reserved.

Mots clés: Cotonnier, stress hydrique, rhizobactérie, Bacillus amyloliquefaciens, fertilisation

\section{Effects of the rhizobacterium Bacillus amyloliquefaciens FZB 42 on the tolerance to water stress as well as on the yield in cotton}

\begin{abstract}
The present work has assessed the potential of the rhizobacterium Bacillus amyloliquefaciens FZB 42 for cotton production. For that, the effect of the rhizobacterium on cotton tolerance to water stress and yield was studied. To determine whether this rhizobacterium can induce resistance to water stress in cotton, 4
\end{abstract}


treatments were tested, namely : i) Seeds dressed in rhizobacterial suspension with soil watering during 15 days, ii) Seeds dressed in rhizobacterial suspension without watering, iii) Seeds dressed in simple water (no rhizobacterium) with watering, and iv) Seeds dressed in simple water (no rhizobacterium) without watering. To test the effect of B. amyloliquefaciens on cotton yield, 6 treatments were compared, namely: i) Conventional mineral fertilization (full dosage) without rhizobacterium, ii) Seeds dressed with rhizobacterial suspension, without mineral fertilization; iii) Seeds dressed with rhizobacterial suspension, plus $10 \%$ mineral fertilization; iv) Seeds dressed with rhizobacterial suspension, plus $25 \%$ mineral fertilization; v) Seeds dressed with rhizobacterial suspension, plus $40 \%$ mineral fertilization; and vi) Seeds dressed with rhizobacterial suspension, plus $50 \%$ mineral fertilization. Results indicated that seeds dressed with B. amyloliquefaciens FZB42 germinated in abundance and faster than untreated seeds; in addition, dressed seeds generated seedlings which have grown faster than those of the control (untreated) regardless of water conditions. The variant treated with rhizobacterium as well as $40 \%$ or $50 \%$ of mineral fertilizer induced cotton yield similar to that of the variant receiving the full fertilizer dosage. We conclude that B. amyloliquefaciens FZB42 can be integrated into cotton production system in order to reduce reliance on mineral fertilizers.

(C) 2018 International Formulae Group. All rights reserved.

Keywords: Cotton, water stress, rhizobacteria, Bacillus amyloliquefaciens, fertilization.

\section{INTRODUCTION}

Le cotonnier, Gossypium hirsutum, est la première plante textile cultivée au monde et constitue plus de $50 \%$ du marché des fibres (Celini, 2001). Plus de deux millions d'agriculteurs cultivent en moyenne un hectare de coton en Afrique de l'Ouest (Martin, 2003). Cette culture fournit plus de $50 \%$ des ressources financières aux populations rurales et par conséquent contribue considérablement à la lutte contre la pauvreté dans les pays producteurs de coton (Martin et al., 2000).

Le cotonnier se cultive au Bénin sur des sols ferrugineux tropicaux et ferralitiques. La pauvreté de ces sols a amené les acteurs à mettre en place un programme de fertilisation minérale qui recommande $200 \mathrm{~kg}$ d'engrais par hectare, chaque année. L'utilisation intensive des engrais minéraux favorise la prolifération des pucerons, et entraîne l'acidification des sols conduisant ainsi à une baisse de leur fertilité (Fayalo et al., 2016 ; Houessou, 2010). Il est donc nécessaire de mettre au point un système de fertilisation respectueux de l'environnement.

Par ailleurs, le changement climatique constitue un problème majeur pour l'agriculture dans de nombreuses régions du monde (Lobell et Gourdji, 2012). La mise au point d'un système de production qui permettrait au cotonnier de résister au stress hydrique est également nécessaire.

RhizoVital ® 421 est un produit naturel contenant des spores de la rhizobactérie Bacillus amyloliquefaciens FZB42, un microorganisme non-pathogène qui existe naturellement dans les sols. Cette rhizobactérie est commercialisée en Europe pour la bio-fertilisation de la pomme de terre et des cultures maraîchères (ABiTEP GmbH, 2015).

La présente étude vise à déterminer l'effet de la rhizobactérie $B$. amyloliquefaciens FZB 42 sur la tolérance au stress hydrique et le rendement du cotonnier.

\section{MATERIEL ET METHODES}

\section{Evaluation de la tolérance au stress hydrique du cotonnier traité avec la rhizobactérie}

Le but de cette étude est de déterminer si B. amyloliquefaciens FZB 42 peut permettre au cotonnier de résister au stress hydrique.

Pour réaliser les tests, les semences du cotonnier (variété H279-1) ont été utilisées. Quatre variantes ont été testées, à savoir :

$>$ semences trempées dans la suspension de la rhizobactérie avec 
arrosage régulier pendant 15 jours (Bacillus arrosé);

$>$ semences trempées dans la suspension de rhizobactérie sans arrosage pendant 15 jours (Bacillus non arrosé) ;

$>$ semences trempées dans l'eau simple (sans rhizobactérie) avec arrosage régulier pendant 15 jours (Témoin arrosé) ;

$>$ semences trempées dans l'eau simple (sans rhizobactérie) ni arrosage (Témoin non arrosé).

Les plants ont été cultivés dans des pots de sept litres de volume. L'essai a été réalisé selon un dispositif expérimental en blocs aléatoires complets (blocs de Fisher) avec 3 blocs composés chacun de 10 pots, soit au total 30 pots pour chaque variante. Nous avons utilisé la souche FZB 42 de la rhizobactérie $\quad B$ amyloliquefaciens (formulation commerciale dénommée "Rhizo Vital") (ABiTEP GmbH, Allemagne). "Rhizo Vital" est un produit contenant 2,5 x $10^{10}$

cfu /ml. Pour réaliser les essais, les semences ont été traitées avec la suspension de rhizobactéries à la concentration de $10^{7}$ spores $/ \mathrm{ml}$. Pour ce faire, $10 \mathrm{ml}$ de 'RhizoVital' ont été dilués dans 1 L d'eau potable. Les semences ont été trempées dans la préparation ainsi obtenue pendant $30 \mathrm{mn}$ puis séchées à l'ombre durant 15 minutes, avant d'être semées aussitôt après. Tous les pots ont été arrosés aussitôt après semis.

Pour garantir les conditions de sécheresse ou d'humidité aux traitements concernés pendant les 15 premiers jours de l'expérimentation, l'essai a été installé en sous-abri. Ce qui a protégé les pots expérimentaux contre la pluie et la rosée.

Pour réaliser les semis, cinq graines ont été mises en terre par pot, pour toutes les variantes.
Les deux variantes ne devant pas connaître de sécheresse (Bacillus arrosé et Témoin arrosé) ont été arrosées tout au long de l'essai. Les deux autres variantes, quant à elles, ont été arrosées seulement à partir du $16^{\text {ème }}$ jour après semis.

L'effet de chaque traitement sur la germination des semences et la croissance des plants a été évalué. Pour ce faire, nous avons d'abord compté le nombre de plants levés par pot au $5^{\text {ème }}$ jour après semis. La taille des plants a été ensuite mesurée au $10^{\text {ème }}, 15^{\text {ème }}$ et $20^{\text {ème }}$ jour après semis à l'aide d'une règle graduée.

\section{Effet de la rhizobactérie combinée avec des doses réduites d'engrais minéraux sur le rendement du cotonnier Site d'expérimentation}

Les essais ont été réalisés sur le site d'expérimentation du centre de recherches agricoles coton et fibres (CRA-CF) d' Okpara (Altitude $320 \mathrm{~m}, 9^{\circ} 18^{\prime} \mathrm{N}, 2^{\circ} 41^{\prime} \mathrm{E}$ ).

Le climat est caractérisé par une saison sèche qui s'étend de novembre à avril-mai et une saison des pluies de juin à octobre. Une température élevée avec une amplitude de $10^{\circ} \mathrm{C}$ et une pluviométrie variant entre 900 et $1300 \mathrm{~mm} / \mathrm{an}$ caractérisent le site. Ce site est également caractérisé par des sols ferrugineux tropicaux.

\section{Application de B. amyloliquefaciens et des différentes doses d'engrais minéraux}

Pour réaliser les essais, les semences ont été traitées avec la suspension de la rhizobactérie à la concentration de $10^{7}$ spores $/ \mathrm{ml}$. Le traitement a été réalisé comme décrit précédemment.

Le dispositif expérimental utilisé est celui du bloc de Fisher avec 6 traitements. Chaque traitement a été répété 6 fois sur des parcelles élémentaires de 6 lignes de $9 \mathrm{~m}$. L'écartement est de $0,80 \mathrm{~m}$ (entre les lignes) 
et de $0,30 \mathrm{~m}$ (entre poquets). Les semis ont été réalisés à 5 graines par poquet et démariés à un plant. Six traitements ont été mis en comparaison :

-Témoin: Semences non trempées dans la suspension de rhizobactérie avec une dose pleine d'engrais minéraux (pratique en vulgarisation).

-Bacillus seul: Semences trempées dans la suspension de rhizobactérie sans apport d'engrais minéraux.

-Bacillus $+10 \%$ Engrais : Semences trempées dans la suspension de rhizobactérie avec un apport de $10 \%$ d'engrais minéraux.

-Bacillus $+25 \%$ Engrais : Semences trempées dans la suspension de rhizobactérie avec un apport de $25 \%$ d'engrais minéraux.

-Bacillus $+40 \%$ Engrais : Semences trempées dans la suspension de rhizobactérie avec un apport de $40 \%$ d'engrais minéraux.

-Bacillus $+50 \%$ Engrais : Semences trempées dans la suspension de rhizobactérie avec un apport de 50\% d'engrais minéraux.

La dose pleine d'engrais recommandée pour

le Nord-Bénin est de $200 \mathrm{~kg} / \mathrm{ha}$ de $\mathrm{N}_{14} \mathrm{P}_{23} \mathrm{~K}_{14} \mathrm{~S}_{5} \mathrm{~B}_{1}$ à 30 jours après semis et 50 $\mathrm{kg} /$ ha d'urée $(46 \% \mathrm{~N})$ à 40 jours après semis.
Les différentes doses d'engrais minéraux apportées sur chaque traitement (en tenant compte des pourcentages de réduction) sont consignées dans le Tableau 1.

Pour la lutte contre les ravageurs, le programme de protection phytosanitaire recommandé pour le Nord-Bénin a été appliqué. Le rendement à l'hectare au niveau des variantes testées a été évalué; pour ce faire, le coton-graine a été récolté sur les deux lignes centrales et le rendement à l'hectare a été calculé.

\section{Analyses statistiques}

Les données ont été analysées avec le progiciel de statistique SPSS 16.0. L'analyse de variance sur mesure répétées, suivant un modèle mixte à deux facteurs (traitements et temps) a été réalisée, afin d'évaluer l'effet de la rhizobactérie sur la germination et la croissance des cotonniers. En ce qui concerne les rendements en coton-graine, le test non paramétrique Kruskal-Wallis test a été réalisé, car les données ne remplissaient pas les conditions d'ANOVA.

Tableau 1 : Dose d'engrais minéraux appliquée dans les traitements mis en comparaison.

\begin{tabular}{lcc}
\hline Traitements & \multicolumn{2}{c}{ Dose en kg/ha } \\
\cline { 2 - 3 } & $\mathbf{N}_{\mathbf{1 4}} \mathbf{P}_{\mathbf{2}} \mathbf{K}_{\mathbf{1 4}} \mathbf{S}_{\mathbf{5}} \mathbf{B}_{\mathbf{1}}$ & Urée (46\% $\mathbf{N})$ \\
\hline Témoin -Semence non trempée & 200 & 50 \\
\hline Bacillus seul & 0 & 0 \\
\hline Bacillus + 10\% Engrais & 20 & 5 \\
\hline Bacillus + 25 \% Engrais & 50 & 12,5 \\
\hline Bacillus + 40 \% Engrais & 80 & 20 \\
\hline Bacillus + 50 \% Engrais & 100 & 25 \\
\hline
\end{tabular}




\section{RESULTATS}

Tolérance au stress hydrique du cotonnier traité avec la rhizobactérie

Le taux de germination des semences au niveau du traitement "Bacillus arrosé" au cinquième jour après semis était de $63 \%$ contre $30 \%$ pour la variante "Témoin arrosé", (sans rhizobactérie, avec arrosage). Pour les pots non arrosés, le taux de germination était de $72 \%$ et $26 \%$, respectivement pour les variantes "Bacillus non arrosé" et "Témoin non arrosé" (Figure 1). Dix jours après semis, le nombre moyen de plantules dans chaque pot était de 3,$66 ; 3,73 ; 2,56$ et 2,23 respectivement pour les variantes "Bacillus arrosé", "Bacillus non arrosé", "Témoin arrosé" et "Témoin non arrosé". En gros, quelles que soient les conditions (stress hydrique ou non), les semences traitées avec la rhizobactérie $B$. amyloliquefaciens FZB42, germent en abondance et plus vite que les semences non traitées. A la fin de l'essai au $20^{\text {ème }}$ jour après semis, le nombre de plantules encore présentes dans chaque pot était de 3,$23 ; 3,20 ; 1,73$ et 2,00 respectivement pour les variantes "Bacillus arrosé", "Bacillus non arrosé", "Témoin arrosé" et "Témoin non arrosé"' (Figure 2). Ceci indique que les semences traitées avec la rhizobactérie donnent naissance à des plantules qui résistent mieux au stress hydrique. L'analyse statistique a également révélé une différence significative entre les variantes traitées avec la rhizobactérie d'une part, et celles non traitées d'autre part $(\mathrm{P}=0,000)$.
La rhizobactérie a également eu un effet sur la croissance des cotonniers. Dans les variantes traitées au Bacillus la taille des plantules était plus grande que celle des variantes non traitées. A la fin de l'essai au $20^{\text {ème }}$ jour après semis, la taille moyenne des plantules était de $139 \mathrm{~mm} ; 131 \mathrm{~mm} ; 106 \mathrm{~mm}$ et $117 \mathrm{~mm}$ respectivement pour les variantes "Bacillus arrosé", "Bacillus non arrosé", "Témoin arrosé" et "Témoin non arrosé" (Figure 3). L'analyse statistique a également révélé une différence significative entre les variantes traitées avec la rhizobactérie d'une part, et celles non traitées d'autre part $(\mathrm{P}=$ $0,000)$.

Effet de la rhizobactérie combinée avec des doses réduites d'engrais minéraux sur le rendement en coton-graine

Le rendement le plus élevé (1892 $\mathrm{kg} / \mathrm{ha}$ ) a été enregistré au niveau de la parcelle témoin (ayant reçu la dose pleine d'engrais minéraux). Ce rendement est suivi de ceux obtenus dans les traitements "Bacillus $+40 \%$ Engrais" et "Bacillus $+50 \%$ Engrais" qui ont donné respectivement des rendements de 1748 $\mathrm{kg} / \mathrm{ha}$ et $1678 \mathrm{~kg} / \mathrm{ha}$ (Figure 4). L'analyse statistique a indiqué qu'il n'y a pas de différence significative entre ces deux variantes d'une part, et le témoin d'autre part $(\mathrm{P}=0,002)$. Les variantes "Bacillus $+40 \%$ Engrais" et "Bacillus $+50 \%$ Engrais" ont donc généré un rendement similaire à celui du témoin ayant reçu la dose pleine d'engrais minéraux.
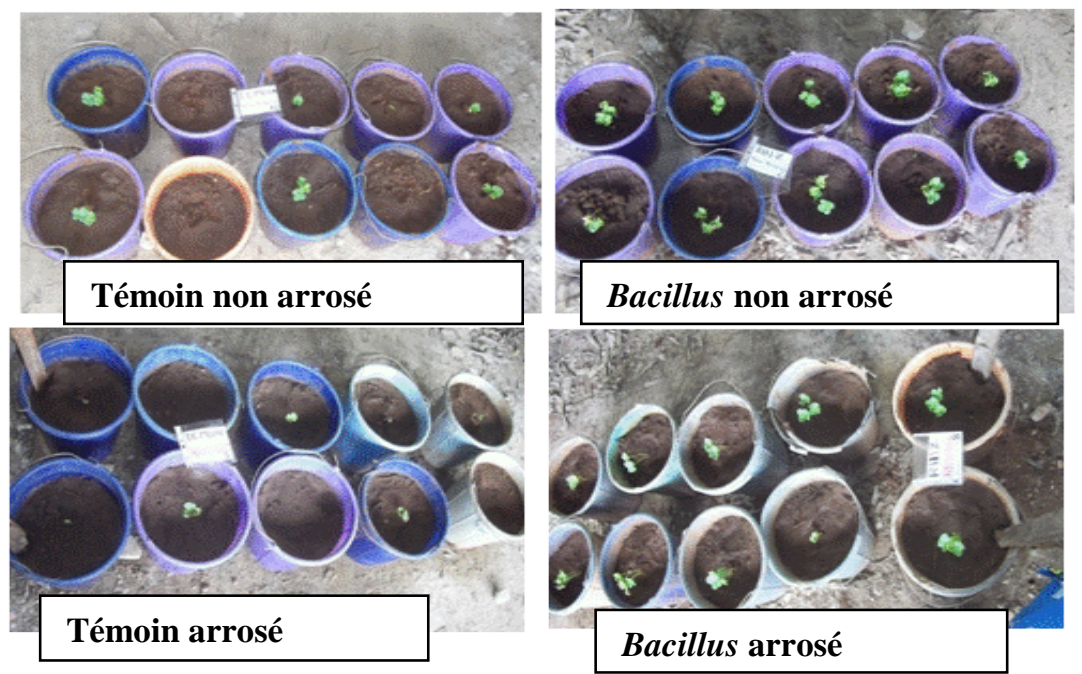

Figure 1: Photo montrant la germination des semences 5 jours après semis 


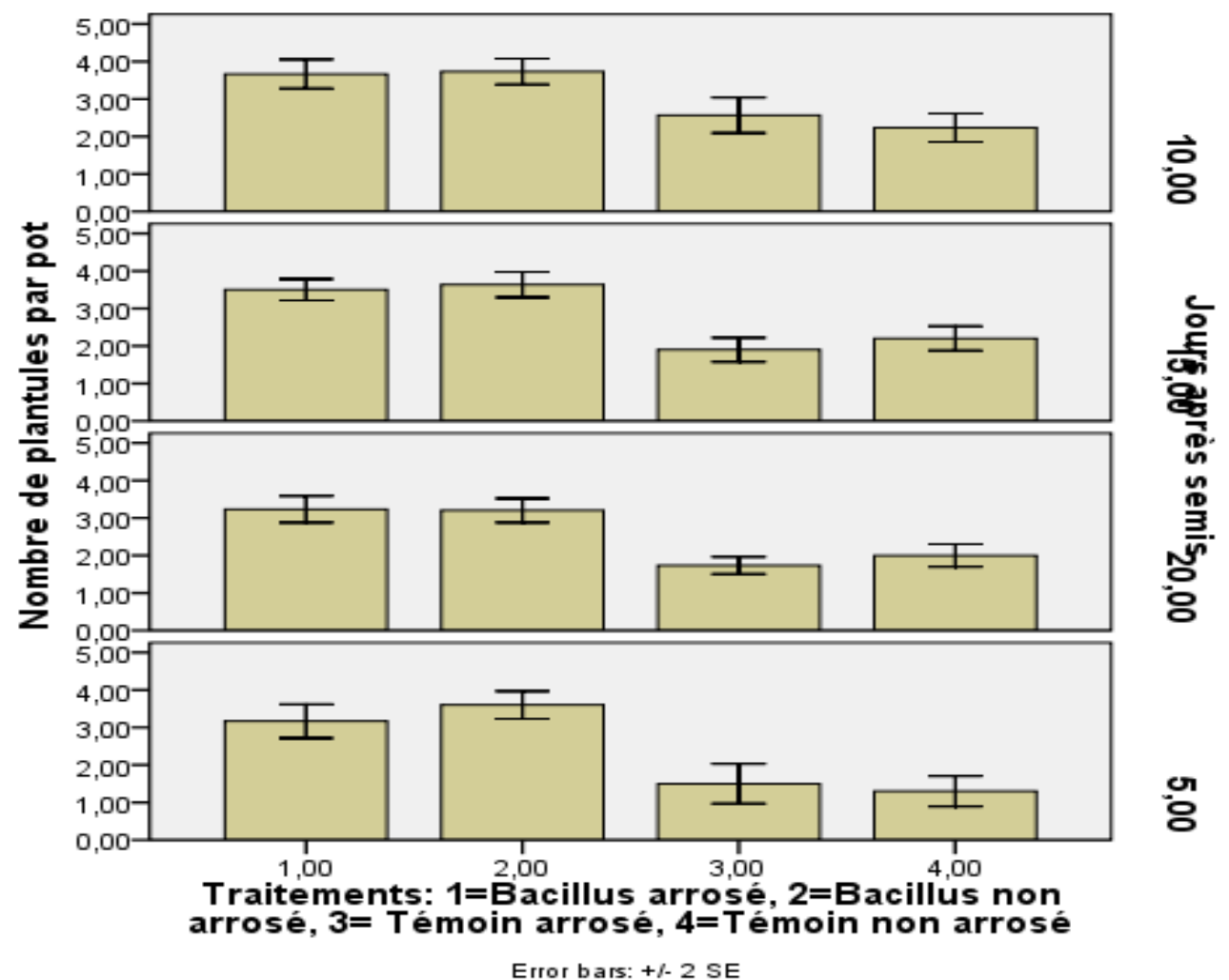

Figure 2 : Nombre moyen de plantules présentes par pot au cours de l'essai sur la tolérance au stress hydrique du cotonnier traité avec la rhizobactérie.

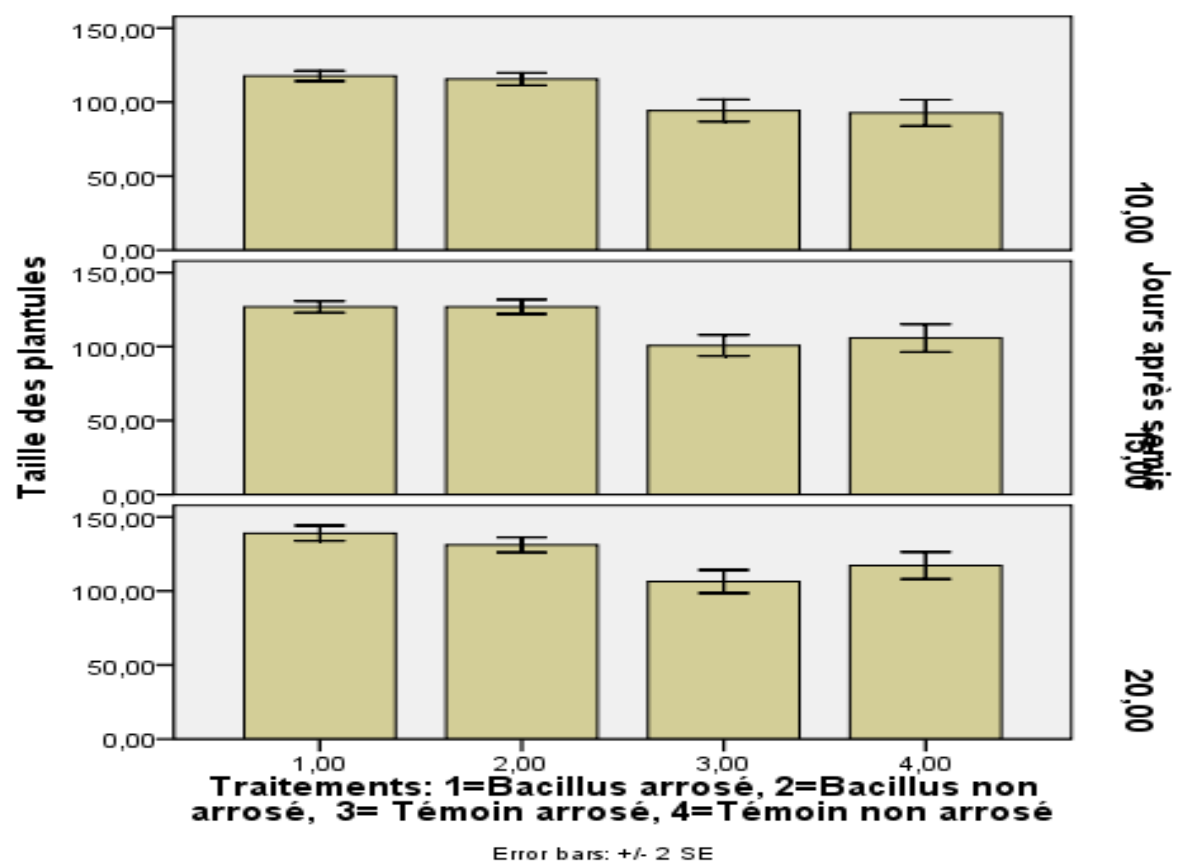

Figure 3: Taille moyenne des plantules au cours de l'essai sur la tolérance au stress hydrique du cotonnier traité avec la rhizobactérie. 


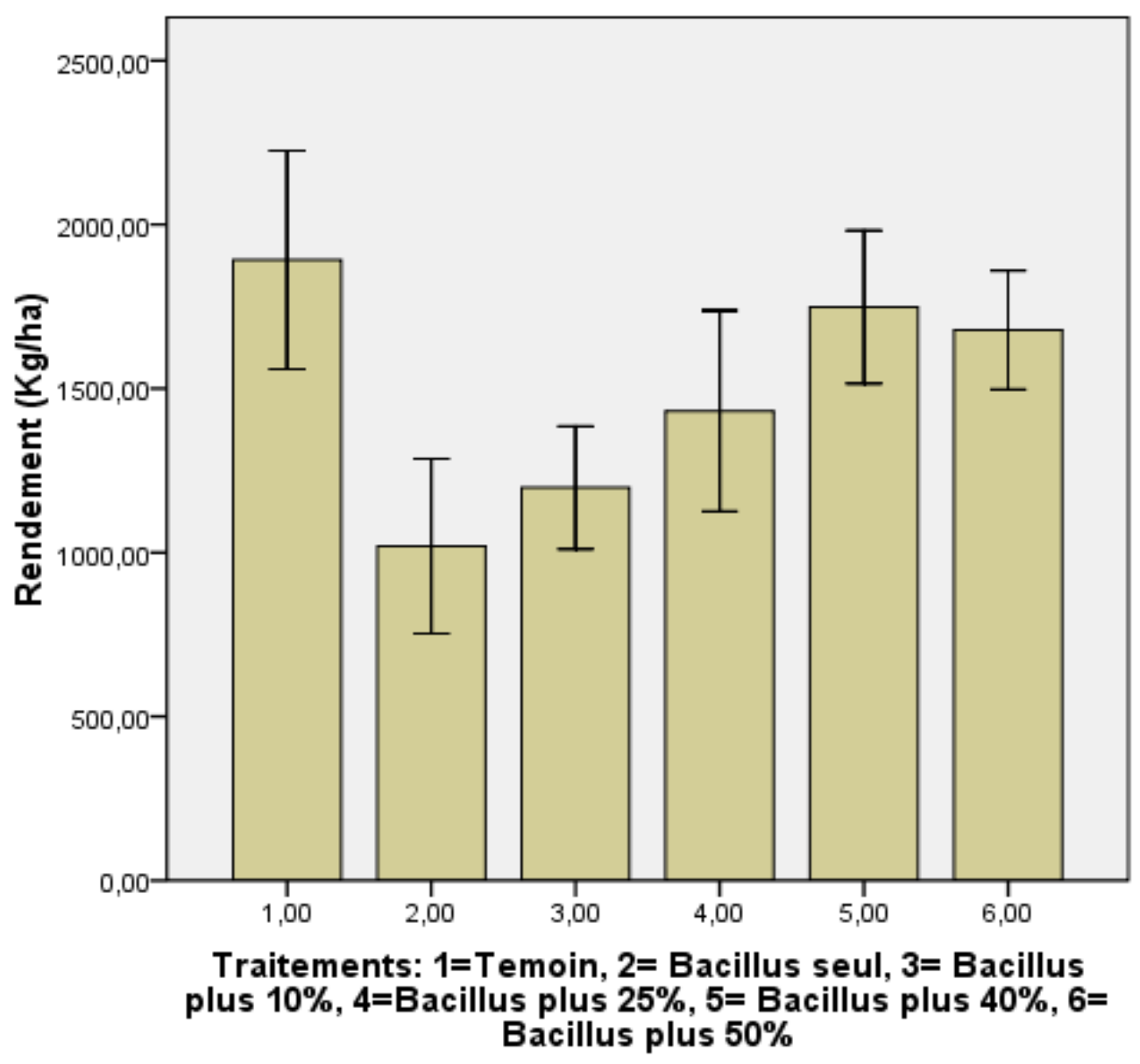

Error bars: +/- $2 \mathrm{SE}$

Figure 4: Rendement en coton-graine dans les différents traitements au cours de l'essai sur la rhizobactérie combinée avec des doses réduites d'engrais minéraux.

\section{DISCUSSION}

Les résultats ont montré que la combinaison de la rhizobactérie $B$. amyloliquefaciens avec des doses réduites de $40 \%$ ou $50 \%$ d'engrais minéraux a permis d'obtenir un rendement en coton-graine similaire à celui du témoin ayant reçu la dose pleine d'engrais minéraux (et non traité avec la rhizobactérie). Les travaux conduits sur le cotonnier au Tadjikistan ont révélé que l'inoculation des semences du cotonnier avec B. subtilis FZB $24 \AA$ a permis d'obtenir un rendement similaire à celui obtenu avec une fertilisation minérale complète (Yao et al., 2006). Aussi, en Egypte, Monir et al. (2012) ont-ils étudié l'effet de la rhizobactérie $B$. amyloliquefaciens FZB42 combinée avec une faible dose d'engrais azoté ; leurs résultats ont indiqué une augmentation du rendement en coton-graine de l'ordre de $75 \%$. En conséquence, nous pouvons conclure que le traitement des semences avec cette rhizobactérie, avec application d'une faible dose d'engrais NPK permet d'obtenir un rendement en coton-graine acceptable.

L'effet bio-fertilisant de cette rhizobactérie s'est également manifesté dans un système de production de coton biologique. En effet, dans une étude visant à comparer l'effet de B. amyloliquefaciens FZB 42 avec 
celui du compost, on a obtenu une augmentation du rendement en coton-graine de l'ordre de $39 \%$ au niveau des plants traités avec la rhizobactérie puis fertilisés au compost (Alavo et al., 2015). On peut donc estimer que B. amyloliquefaciens FZB 42 peut être utilisée dans tous les systèmes de production cotonnière. De nombreux auteurs ont également démontré que l'inoculation des semences avec des rhizobactéries promotrices de la croissance des plantes se solde par une augmentation considérable du rendement (Suslow, 1982 ; Hernandez et al., 1995 ; Nezarat et Gholami, 2009 ; Adjanohoun et al., 2012).

Le bon niveau de rendement en cotongraine obtenu au niveau des plants traités avec la rhizobactérie, en dépit de la réduction de la dose d'engrais minéraux, peut s'expliquer par différents facteurs. En effet, l'un des modes d'action de Bacillus spp. est la colonisation de la rhizosphère avec production de molécules antibiotiques; ce qui empêche les champignons phytopathogènes de faire pourrir les semences mises en terre (Krebs et al., 1998 ; Zimmer et al., 1998; Kilian et al. 2000; Kloepper et al., 2004; Idriss et al., 2007). Ce mécanisme peut également expliquer le fort pouvoir germinatif observé au niveau des semences traitées avec la rhizobactérie au cours de nos différentes investigations. Il y a également la promotion de la croissance végétale et radiculaire induite par ces rhizobactéries. En effet, on a pu constater in vitro, sous l'effet de B. subtilis, la formation de substances et de mélanges de substances ayant une action analogue à celle de la cytokinine ou de l'auxine. C'est-à-dire que l'augmentation du volume et des ramifications $\mathrm{du}$ système racinaire modifie le bilan phytohormonal endogène des plantes. En effet, l'appareil radiculaire plus développé permet une meilleure absorption de l'eau et des substances nutritives, et par conséquent une croissance plus rapide et une plus grande tolérance au stress hydrique (Kilian et al., 2000). Des études réalisées sur d'autres souches de rhizobactérie par différents auteurs indiquent également que les rhizobactéries promotrices de la croissance des plantes confèrent à ces dernières la capacité de résister au stress hydrique et à la chaleur (Timmusk et Wagner, 1999; Dimkpa et al., 2009; Yang et al., 2009 ; Kassim et al., 2013 ; Bresson, 2013; Bresson et al., 2013 ; Abd El Daim et al., 2014). Ces différents modes d'action peuvent donc expliquer les résultats que nous avons obtenus avec $B$. amyloliquefaciens FZB42 au cours de la présente étude.

\section{Conclusion}

L'inoculation des semences du cotonnier avec la rhizobactérie $B$. amyloliquefaciens FZB 42 est une technologie qui pourrait permettre aux producteurs de garantir une bonne densité des plants et par ricochet un bon rendement en coton-graine, tout en réduisant la dépendance aux engrais minéraux.

\section{CONFLIT D'INTÉRÊT}

Nous déclarons n'avoir pas de conflit d'intérêt.

\section{CONTRIBUTIONS DES AUTEURS}

Les auteurs apparaissent dans l'ordre de leur taux de contribution, le premier auteur ayant fait la plus grande contribution. BCTA a conçu le projet, a supervisé les travaux, a analysé les résultats et a rédigé le manuscrit. MA et LD ont collecté les résultats sur l'effet de la rhizobactérie combinée avec des doses réduites d'engrais minéraux et GDF a surtout travaillé sur tolérance au stress hydrique.

\section{REMERCIEMENTS}

Nous remercions très sincèrement Prof. Em. Dr. Helmut Bochow pour son assistance technique et pour avoir fourni gratuitement les échantillons de la rhizobactérie. $\mathrm{La}$ participation des ouvriers du CRA-CF aux travaux champêtres est grandement appréciée.

\section{REFERENCES}

Abd El-Dam IA, Bejai S, Meijer J, Joahn M. 2014. Improved heat stress tolerance of wheat seedlings by bacterial seed 
treatment. Plant Soil, 379: 337-350. DOI 10.1007/s11104-014-2063-3

ABiTEP GmbH. 2015. RhizoVital ® 421. Bacillus amyloliquefaciens FZB42 liquid a plant growth-promoting rhizobacteria. http://www.abitep.de. Consulté le 27/03/2015.

Adjadohoun A, Noumavo PA, Sikirou R, Gotoechan-Hodonou H, Yehouenou B, Baba-Moussa L. 2012. Effets des rhizobactéries PGPR sur le rendement et les teneurs en macroéléments du maïs sur sol ferralitique non dégradé au SudBénin. Int. J. Biol. Chem. Sci., 6(1): 279288.

Alavo TBC, Boukari S, Fayalo DG, Bochow H. 2015. Cotton fertilization using PGPR Bacillus amyloliquefaciens FZB42 and compost: Impact on insect density and cotton yield in North Benin, West Africa. Soil \& Crop Sciences. Doi:10.1080/23311932.2015.1063829.

Bresson J, Varoquaux F, Bontpart T, Touraine B, Vile D. 2013. The PGPR strain Phyllobacterium brassicacearum STM196 induces a reproductive delay and physiological changes that result in improved drought tolerance in Arabidopsis. New Phytologist, 200(2): 558-569.

Celini L. 2001. Le Puceron du cotonnier : Aphis gossypii (Glover) et son parasite Aphelinus gossypii Timberlake en République Centrafricaine, Insect $\mathrm{n}^{\circ}$ 122-2001(3) : 6- 10.

Dimkpa C, Weinand T, Asch F. 2009. Plantrhizobacteria interactions alleviate abiotic stress conditions. Plant Cell Environ, 32:1682-1694.

Fayalo GD, Aouco A, Alavo TBC. 2016. Dynamique des populations du puceron Aphis gossypii (Homoptera: Aphididae) sur le cotonnier en conditions de fertilisation minérale et rhizobacteriologique. Tropicultura, 34(1) : 98-104.
Hernandez AN, Hernandez A, Heydrich M. 1995. Seleccion de rhizobacterias asiciadas al cultivo del maiz. Cultivos Tropicales, 16(3): 5-8.

Houessou HG. 2010. Contribution à la lutte contre le puceron (Aphis gossypii G.) parasite d'Ocimum Gratissimum L. en régions urbaines au sud du Bénin. Mémoire de Master en Entomologie Appliquée, FAST/UAC. 39 p.

Idriss EE, Iglesias DJ, Talon M, Borriss R. 2007. Tryptophan-dependent production of indole-3-acetic acid (IAA) affects level of plant growth promotion by Bacillus amyloliquefaciens FZB42. MPMI 20: 619-626.

Kassim WA, Omar MN, Abd El-Daim IA, Bejai S, Meijer J. 2013. Control of drought Stress in weat usinig Plant Growth-Promoting Bacteria. J. Plant Growth Regular, 32: 122-130.

Kilian M, Steiner U, Krebs B, Junge H, Schmiedeknecht G, Hain R. 2000. FZB24® Bacillus subtilis - mode of action of a microbial agent enhancing plant vitality. Pflanzenschutz-Nachrichten Bayer 1/00, 1: 72-93.

Kloepper JW, Ryu CM, Zhang S. 2004. Induced systemic resistance and promotion of plant growth by Bacillus spp. Phytopathol., 94: 1259-1266.

Krebs B, Hoding B, Kubart S, Alemayehu M, Junge H, Schmiedeknecht G, Grosch R, Bochow H, Hevesi M. 1998. Use of Bacillus subtilis as biocontrol agent. I. activities and caraterization of Bacillus subtilis strains. Z. pflanzenkh. Pflanzenshutz, 105: 181-197.

Lobell DB, Gourdji SM. 2012. The influence of climate change on global crop productivity. Plant Physiol, 160:1686 - 1697.

Martin T, Ochou G, Hala-N'klo F, Vassal JM, Vaissayre M. 2000. Pyrethroïd resistance in the cotton bollworm, 
Helicoverpa armigera (Hubner) in west Africa. Pest management Sc., 56(6): 549-554.

Martin T. 2003. La résistance aux insecticides de Helicoverpa armigera (Hübner) en Afrique de l'Ouest: du mécanisme à la gestion. Thèse de Doctorat de 1'Université Toulouse III, discipline chimie. pp: 1-80.

Monir MEH, Bochow H, Junge H. 2012. The biofertilising effect of seed dressing with PGPR Bacillus amyloliquefasciens FZB 42 combined with two levels of mineral fertilizing in African cotton production, Archives of Phytopathology and Plant Protection : $1-11$ DOI : 10.1080/03235408.2012.673259.

Nezarat S, Gholami A. 2009. Screening plant growth promoting rhizobacteria for improving seed germination, seedling growth and yield of maize. Park. J. Biol. Sci., 12(1): 26-32.

Suslow TV, Schroth MN. 1982. Rhizobacteria of sugarbeet: Effects of seed application and root colonization on yield. Phytopathology, 72:199-206.

Timmusk S, Wagner EGH. 1999. The plantgrowth-promoting Rhizobacterium Paenibacillus polymyxa induces changes in Arabidopsis thaliana gene expression: a possible connection between biotic and abiotic stress responses. Mol PlantMicrobe Interact., 12(11): 951-959.

Yang J, Kloepper JW, Ryu CM. 2009. Rhizosphere bacteria help plants tolerate abiotic stress. Trends Plant Sci., 4:1-4.

Yao AV, Bochow H, Karimov S, Boturov U, Sanginboy S, Sharipov AK. 2006. Effect Of FZB 24® Bacillus subtilis as Biofertilizer On Cotton Yields In Field Tests. Archives of Phytopathology and Plant Pathology, 39(4): 323-328.

Zimmer J, Issoufou I, Schmiedeknecht G, Bochow H. 1998. Population dynamics of Bacillus subtilis as biocontrol agent Under controlled conditions. Med. Fac. Lan, 29: 17-26. 